\title{
Interaction Analysis of MAT Foundation and Space Frame for the Non Linear Behaviour of the Soil
}

\author{
Dr.D. Daniel Thangaraj and Dr.K. Ilamparuthi
}

\begin{abstract}
Structural analysis is one of the most important aspects in structural design, since it gives an idea about the performance of the structure under load. Right from the beginning when the process of development started in the field of soil-structure interaction, an attempt has been made to bring sophistication in the theoretical methods of analysis. In the conventional analysis the stress-strain response of the soil is not considered. The equilibrium equations are satisfied but the compatibility is not considered. But in reality the soil, the foundation and the frame act together as a single compatible unit. The non-linear response of soil is considered for this analysis. The non-linear response of the soil is included in the analysis through multi linear isotropic (MISO) model and the parameters used in the parametric study are relative stiffness factors krs and ksb which are the function of the modulus of the soil, modulus of frame raft materials and geometric properties of the structural elements.
\end{abstract}

Keywords--- Contact Pressure, Interaction, MAT, NonLinear, Settlement and Moment

\section{INTRODUCTION}

$\mathrm{T}$ HE subject of the soil-structure interaction has attracted the attention of both structural and geotechnical engineers all over the world. Their major concern is the analysis and the design of a variety of structures, namely multi-storeyed buildings, towers, chimneys, industrial-structures, reactors and buried structures. The design of these structures undoubtedly represents one of the most difficult technical aspects of civil engineering practice, as it requires a synthesis of structural and geotechnical analyses.

In the recent past, construction industry has witnessed a dramatic growth particularly in the construction of multistoreyed buildings because of rapid urbanization and industrial development. Buildings of moderate heights are constructed more in numbers and their growth rate increases year after year. Till recently buildings were designed based on greatly simplified assumptions combined with rudimentary analysis (conventional method). However, designers have realized that safe and economical design of a structure cannot be thought of unless interaction among the soil, the foundation and the structure is taken into account. Therefore, the interaction

Dr.D. Daniel Thangaraj, Assistant Professor of Civil Engineering, Anna University College of Engineering Tindivanam-604 001. Email:danielthangarajd@gmail.com

Dr.K. Ilamparuthi, Professor and Head, Department of Civil Engineering, Anna University, Chennai-25. South India. analysis between the soil, the foundation and the superstructure is very essential to predict the correct forces in the columns and the beams of the superstructure.

In this paper a framed structure of 3 bay x 5 bay supported on a foundation system known as mat is considered to evaluate the influence of thickness of mat and nonlinear behavior of the soil on forces and deformation of the frame. Based on the analyses of the soil- mat-space frame system, the settlement is higher for the non-linear soil condition. The magnitude of the contact pressure is maximum at the corner of the raft. Bending moment in the raft, the influence of ksb is appreciable in the end bay moments.

\section{LITERATURE REVIEW}

The present discussion considers the development of the interaction analysis taking into account the influence of the non-linear behavior of the soil. Each element of the system is considered to have linear stress-strain characteristics. The non-linearity of the behavior of the soil can be taken into account by the well-developed incremental analysis. In this case, however, it is necessary to represent the soil by finite elements, and the use of the Boussinesq type of continuum representation is restricted to a uniform soil layer.

Meyerhof [1] showed the importance of the rigidity of the superstructure in the design of the foundation through a simple interaction analysis. He developed an approximate method to estimate the stiffness of the superstructure and to relate it to the deformation of the soil. Applicability of the proposed method was demonstrated through an analysis of a frame on London clay which showed that a relatively small differential settlement of footing induced large forces and moments on all structural members, particularly at external joints in the lower storey.

He calculated the secondary moments due to the settlement of the footing by the slope deflection method and distributed them using the Hardy Cross method. He also suggested an approximate method for the estimation of the moments in members of the frame supported on isolated footings. Gorbunov et al [2] introduced an approximate method for studying the behavior of beams and plates founded on a elastic half space. The unknown distribution of the contact pressure, the settlement and the bending moment were expressed as double power series. On the basis of the bi-harmonic equation of bending of slab and edge conditions the unknown coefficients were obtained as an infinite linear system. Solutions were obtained for the settlement of a rigid foundation, the bending of a flexible slab under uniformly distributed loads and concentrated loads. Wardle and Fraser 
[3] investigated the effect of a finite soil layer with a linearly increasing modulus of deformation (or any other variation which can be substituted by a finite number of thin layers) using programme FOCALS. Wardle and Fraser developed a programme for the analysis of plates on layered elastic foundations. Witt [4] analyzed the square plate founded on a homogeneous soil and compared it with a non-homogeneous soil by using FEM. The heterogeneity of the foundation caused by the orthotropic was taken into account when forming the equations of equilibrium and variable thickness of the foundation by approximating it by Heir's functions. The results obtained for the heterogeneous condition were compared with those obtained for the homogenous condition. It was found that the deflection of the foundation and the plate differ in extreme cases up to $10 \%$ with the discrepancy of the bending moment up to $60 \%$.

Wang et al [6] (2000) studied the behavior of a plate on a non-homogeneous elastic half space by FEM. The interaction between the plate and the soil was studied numerically by the isoparametric element method and it yielded more accurate results. A number of examples for plates on the nonhomogeneous foundation have also been analyzed. Ball et al [5] analysed a raft foundation founded on the Winkler medium using FEM with the help of a computer. He made the following assumptions: (i) the raft acts as an isotropic homogeneous elastic solid, (ii) the subgrade reactions are vertical vectors and are proportional to the deflection of the node and (iii) the subgrade reaction is equal to the spring constant at a node multiplied by the deflection of that node. They included the effect of the superstructure in the analysis of the raft and calculated the settlement and the contact pressure below the raft.

King and Chandrasekaran [7] formulated a finite element procedure and analyzed a plane frame supported on a combined footing in which the frame and the combined footing were discretised into beam bending elements and the soil mass into plane rectangular elements. A zero thickness friction element was also adopted for the raft-soil interface. This element was, however, useful only in the presence of the lateral loads. They developed a semi-analytical method to study the effect of the rigidity of the superstructure on the performance of the foundation. Bhandari and Rao [8] in their paper on "Concept of rigidity in foundation analysis" brought out the contribution of the modulus of deformation of the soil, the thickness of the foundation slab and the stiffness of the superstructure to the relative rigidity factor through experimental and analytical works. Srinivasaraghavan [9] studied the interaction among structure, raft and soil and introduced the term 'relative rigidity index' (RRI) to show the combined effect of the rigidity of the superstructure, the raft and the soil. He analysed 2 bay $\times 2$ bay on raft foundation and 2 bay $\times 4$ bay frames on raft and frames on individual footings. Brown et al [10] examined the effect of the sequence of construction on the interaction behavior and found that the effective stiffness of a building during construction is about half the stiffness of the completed structure. Hora and Sharm [11] analyzed a plane frame of two storey two bay in filled frame-foundation beam soil system considering linear elastic behavior of the superstructure (including in-fill). The elasto- plastic interaction analysis has been carried out considering the subsoil to yield according to various yield criteria. The results of the elasto plastic interaction analysis are compared with conventional and linear elastic interaction analysis. They concluded that, the collapse load for the in filled frame foundation beam soil system is marginally less compared to plane frame foundation beam soil system. It is due to the fact that the inclusions of in-filled panels significantly increased in the stiffness of superstructure, which has marginal reduction in differential settlement of the foundation beam. Very recently Swamy et al [13] carried out uncoupled and coupled FE analyses to study the interface characteristics between the soil and isolated footing on structure. In this study two independent cases were considered to represent smooth and rough interface. In the first case the smooth interface was modeled as a complete slip and in the second case rough interface was modeled by assigning complete welding between the joints and foundation and soil elements. The FE code developed for these two conditions were applied to evaluate the influence of interface elements in soil structure interaction analysis of structures supported by isolated footings.

From the above discussion, it is clear that most of the previous investigators have analyzed the mat either founded on an elastic half-space or wherein the soil was treated as a linear elastic solid. The settlement, the bending moment, and the contact pressure on the raft were analyzed in detail but the modulus of the soil and thickness of the raft was not discussed for the non-linear behavior of the soil. Hence in this paper to find the effect of thickness of the mat and non-linear soil on the behavior of mat and frame are studied.

\section{Details of THE PROBLem}

The plan of the quarter raft and the position of the columns of 3 bay $\times 5$ bay frames are shown in Figure1. The spacing between the columns is $6 \mathrm{~m}$ and the column height between the floors is $3.5 \mathrm{~m}$. In the analysis, the stiffness of the wall and the slab are not included. The load on the slab including self-weight and weight of the wall are considered and applied as uniformly distributed loads on the beams. The influence of these two parameters on the forces and moments in superstructure and the mat were studied. Analyses were carried out for the following $\mathrm{k}_{\mathrm{rs}}=0.001,0.005$ and 0.01 and $\mathrm{k}_{\mathrm{sb}}$ $=15,20,30,60$ and 100. The geometric properties of the frame and the material properties adopted in the analysis are presented in Table 1.

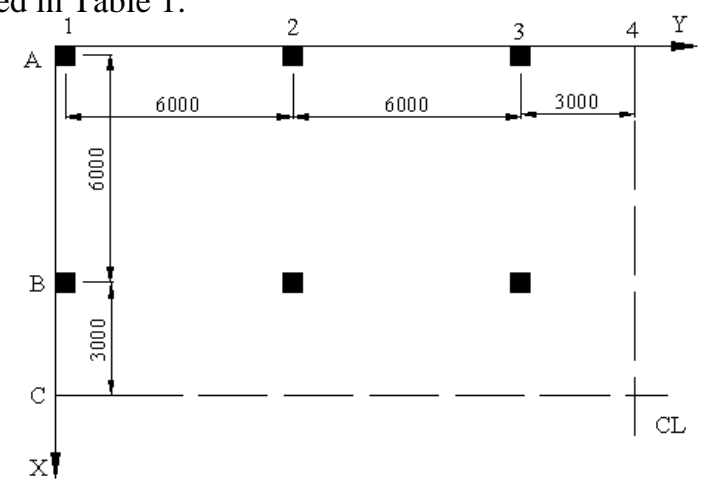


Figure 1: Plan of Quarter Raft and Column Position (All Dimension are in $\mathrm{mm}$ )

Table 1: Geometric and elastic properties of frame and raft and soil

\begin{tabular}{|l|l|l|}
\hline \multirow{2}{*}{$\begin{array}{l}\text { Column } \\
\text { size, } \mathrm{m}\end{array}$} & Storey- 1,2,3 & $0.5 \times 0.5$ \\
\cline { 2 - 3 } & Storey- 4, 5 & $0.4 \times 0.4$ \\
\hline Beam size, $\mathrm{m}$ & $0.3 \times 0.6$ \\
\hline Modulus of concrete, $\mathrm{kPa}$ & $2.5 \times 10^{7}$ \\
\hline Load on inner beams & $35 \mathrm{kN} / \mathrm{m}$ \\
\hline Load on outer beams & $28 \mathrm{kN} / \mathrm{m}$ \\
\hline Poisson's ratio of concrete & 0.15 \\
\hline Poisson's ratio of soil & 0.35 \\
\hline Modulus of soil, MPa & 20 to 134 \\
\hline
\end{tabular}

The non-linear response of the soil is included in the analysis through MISO model (multi linear isotropic) and the parameters used in the parametric study are relative stiffness factors $\mathrm{k}_{\mathrm{rs}}$ and $\mathrm{k}_{\mathrm{sb}}$ which are the function of the modulus of the soil, modulus of frame raft materials and geometric properties of the structural elements. The non-linear relation between stresses and strains of the soil adopted in the analysis is as shown in Figure 2.

Figure 2: Stress-strain curve for the cohesion less soil

\section{RELATIVE STIFFNESS}

The interaction between the frame and the raft depends on

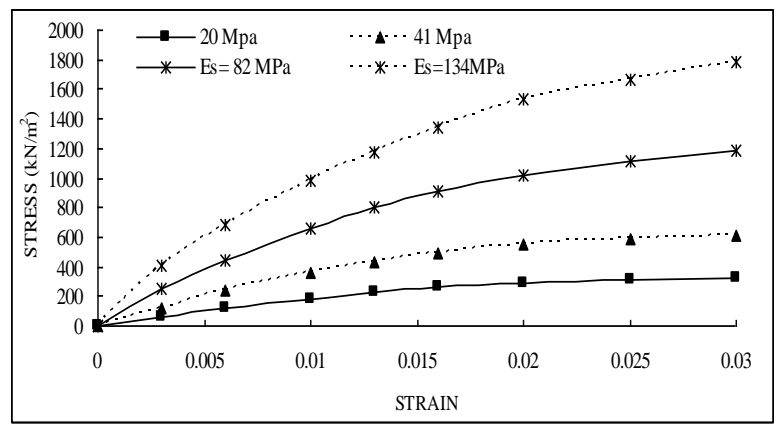

the relative stiffness of the raft and the frame, which are the ratios of the absolute stiffness of the frame $\mathrm{k}_{\mathrm{b}}$, the foundation $\mathrm{k}_{\mathrm{r}}$, and the soil $\mathrm{k}_{\mathrm{s}}$. The relative stiffness $\mathrm{k}_{\mathrm{sb}}$ and $\mathrm{k}_{\mathrm{rs}}$ are determined based on the recommendation of Brown and $\mathrm{Yu}$ which are as follows.

$$
\begin{aligned}
& k_{s b}=\frac{E_{s} l^{4}}{m E_{b} I_{b}\left(1-v_{s}^{2}\right)} \\
& k_{r s}=\frac{16 E_{r} I_{r}\left(1-v_{s}^{2}\right)}{\pi E_{s} L^{4}}
\end{aligned}
$$

where, $\mathrm{m}=$ Number of storeys, $\quad \mathrm{E}_{\mathrm{b}}=$ Elastic modulus of beam, Es = Elastic modulus of soil, $\mathrm{E}_{\mathrm{r}}=$ Elastic modulus of raft, $I_{b}=$ Moment of Inertia of beam, $I_{r}=$ Moment of Inertia of raft, $L=$ Length of the raft, $1=$ Span of the beam, and $\mu_{\mathrm{s}}=$ Poisson's ratio of soil.

\section{FINITE ELEMENT MODEL}

The complexities involved in the interaction analysis of the mat and the superstructure can be simplified to a larger extent if the finite element technique is used.

\subsection{Frame Model}

The frame is modelled as an assemblage of beam elements (Beam4). Beam4 is a uniaxial element which has the capabilities of tension, compression, torsion and bending capabilities. This element has two nodes and six degrees of freedom at each node and it is treated as Timoshenko beam elements. They are translations in the nodal $\mathrm{x}, \mathrm{y}$, and $\mathrm{z}$ directions and rotations about the nodal $\mathrm{x}, \mathrm{y}$, and $\mathrm{z}$ directions. The joints between the columns and beams are assumed to be rigid.

\subsection{MAT Foundation and Contact Element}

The mat is modelled as a plate-bending element (Shell93) with eight nodes having six degrees of freedom at each node. The moment per unit length of the mat is calculated in the element co-ordinate system. The interface characteristics between the mat and the soil are represented by the combination of element Targe170 and Conta174. These interface elements are adopted to represent contact of dissimilar materials of soil and concrete mat as adopted by other researchers [14]. The interface adopted in this study represents no slip between soil and foundation.

\subsection{Soil Model}

The soil is treated as an isotropic, homogenous and elastic half space medium. For the linear analysis, the initial tangent modulus $\left(\mathrm{E}_{\mathrm{s}}\right)$ and Poisson's ratio $\left(\mathrm{v}_{\mathrm{s}}\right)$ are the inputs. The soil medium below the mat is modelled using the eight-node brick element (SOLID45) having three degrees of freedom of translation in the $\mathrm{x}, \mathrm{y}$ and $\mathrm{z}$ directions at each node. In order to find the extent of the soil region to be used in the study, many trial analyses were carried out and was found that for the width and the thickness of the soil medium more than 2.5 times the least width of the mat foundation the variation in settlement and the contact pressure was negligible, thus the region of soil medium considered was 2.5 time the width in all three direction by arresting vertical translation at the bottom boundary and arresting lateral translation on the vertical boundaries. Fine meshes with aspect ratio 1 are generated close to the mat while meshes generated away from the mat area are made coarser gradually.

\section{RESULTS AND DISCUSSION}

A rectangular mat of $18 \mathrm{~m}$ x $30 \mathrm{~m}$ supporting 24 columns spaced at $6 \mathrm{~m}$ centre to centre on both the directions was analyzed for the linear and non-luinear behavior of the soil. The results of the analyses are presented and discussed below. 


\subsection{Settlement of the Raft}

Figure 3 shows the variation of normalized settlement, w/l (w- settlement, 1 = span) along the section B1-B4 for various $\mathrm{k}_{\mathrm{sb}}$ values. In general, the settlement at the edge of the raft is marginally higher than that at the centre irrespective of the $\mathrm{k}_{\mathrm{sb}}$ value. However, the settlement along the section B1-B4 is decreased appreciably with increase in the $\mathrm{k}_{\mathrm{sb}}$ value and the difference in settlement between the points B1 and B4 is also less. The maximum reduction in the differential settlement between $\mathrm{k}_{\mathrm{sb}}=15$ and 100 along this section is $86 \%$ for $\mathrm{k}_{\mathrm{rs}}=$ 0.001 . Similarly the maximum reduction in differential settlement between $\mathrm{k}_{\mathrm{sb}} 15$ and 100 along this section is $87 \%$ for $\mathrm{k}_{\mathrm{rs}}=0.01$. In the case of non-linear conditions the differential settlement of the raft is almost negligible i.e the settlement is almost uniform irrespective of the $k_{\mathrm{rs}}$ and $\mathrm{k}_{\mathrm{sb}}$ values. The difference in the settlement between the linear and non-linear conditions is compared in Figure 4 for the centre and the corner points of the raft. At these two points the settlement is higher for the non-linear soil condition irrespective of $\mathrm{k}_{\mathrm{rs}}$ and $\mathrm{k}_{\mathrm{sb}}$ values. As $\mathrm{k}_{\mathrm{sb}}$ increases, the difference in the settlement decreases both at the centre and the corner of the raft and the difference become almost negligible for the higher values of $\mathrm{k}_{\mathrm{sb}}(100)$ and $\mathrm{k}_{\mathrm{rs}}(0.01)$. However maximum difference is for the $\mathrm{k}_{\mathrm{rs}}$ value of 15 and is around $62 \%$ and $48 \%$ for the corner and centre points of the raft. In Figure 5 the differential settlement is presented in the form of the angular distortion, which is defined as the ratio of the differential settlement to the spacing between the column A1 (corner column) and B3 (column close to centre of raft). If $\mathrm{k}_{\mathrm{rs}}$ is higher, the differential settlement is lesser and it decreases with increase in $\mathrm{k}_{\mathrm{sb}}$. This is another evidence for the reduction in the settlement of the raft due to increase in the modulus of the soil and the thickness of the raft.

\subsection{Contact Pressure below the Raft}

The contact pressure distribution is presented in figure 6 for the longitudinal sections A1-A4 and B1-B4 of the raft. It is seen from the figure that the contact pressures at the edges are higher than that at the center. But the magnitude of the contact pressure is maximum at the corner of the raft. In the corners, the concentration of the contact pressure is around 11 times the average pressure, $\mathrm{q}(\mathrm{q}=$ total vertical load / area of the raft). Similarly, at the edge (ie along the periphery) the same is 4.5 times the q value. But they act over a very smaller area and drastically get reduced just away from the edge and the corner points of the raft. The contact pressure at the centre part of the raft is uniform with the magnitude and found to vary between $0.7 \mathrm{q}$ and $1.0 \mathrm{q}$.

The contact pressure distribution is presented in Figure 7 for the longitudinal sections A1-A4 and B1-B4 of the raft for the non-linear behavior of soil. It is seen from the figure that the contact pressures at the edges are higher than that at the center. But the magnitude of the contact pressure is maximum at the corner of the raft. This variation is far different from what we normally adopt in the conventional design of the raft foundation (i.e. distribution is uniform throughout the area of the raft). In the corners, the concentration of the contact pressure is around 2.0 times the average pressure, $\mathrm{q}(\mathrm{q}=$ total vertical load / area of the raft). At the edge B1 the same is 1.5 times the $\mathrm{q}$ value. But the contact pressure is reduced drastically just away from the edge and the corner points of the raft. The contact pressure at the centre part of the raft is found to vary between $1.4 \mathrm{q}$ and $0.9 \mathrm{q}$. Similarly, the contact pressure of the raft at the centre and the corner for the linear is shown in Figure 8 for various $\mathrm{k}_{\mathrm{rs}}$ and $\mathrm{k}_{\mathrm{sb}}$ combinations at the centre and the corner of the raft. For the values of $\mathrm{k}_{\mathrm{rs}}$ (0.001 and 0.01) and $\mathrm{k}_{\mathrm{sb}}(15$ and 100) shown in the figure, the contact pressure at the centre part of the raft along B1-B4 is 0.8 times the average pressure. The influence of $\mathrm{k}_{\mathrm{sb}}$ on the contact pressure is almost nil. But the contact pressure decreases with the increase in $\mathrm{k}_{\mathrm{rs}}$ particularly at the column points and the distribution becomes more uniform over most parts of the raft except at the edges and the corners. The pattern of the contact pressure obtained from the FEM analysis showed a higher edge pressure and it decreases towards the centre of the raft.

\subsection{Bending Moment in the Raft}

Bending moments in the raft are analysed by varying the non dimensional parameters $\mathrm{k}_{\mathrm{sb}}$ and $\mathrm{k}_{\mathrm{rs}}$ and the typical variation in the bending moment along the section A1-A4 for the $\mathrm{k}_{\mathrm{rs}}$ values of 0.001 is shown in Figure 9. The influence of $\mathrm{k}_{\mathrm{sb}}$ is appreciable in the end bay moments. The span moments increases with an increase in $\mathrm{k}_{\mathrm{sb}}$ at both the sections and the maximum increase is in the end bay of the section A1-A4 which is about $33 \%$.

\subsection{The Bending Moment in the Columns}

The effects of the relative stiffness of the frame and the raft on the moments of column A1 and B1 are represented in Figure 10 since the variation in moment due to change in the interaction parameters is more in these two columns. The moment in both the columns at the first floor level has found to decrease with an increase in $\mathrm{k}_{\mathrm{sb}}$ for the $\mathrm{k}_{\mathrm{rs}}$ values of 0.001 and 0.01 . In the columns at the other floors the variation is marginal (increase or decrease) irrespective of the $\mathrm{k}_{\mathrm{sb}}$ and $\mathrm{k}_{\mathrm{rs}}$ values. However, the decrease in the moment of the first floor column is high for $\mathrm{k}_{\mathrm{rs}}=0.001$ and the reduction is $65 \%$ and $70 \%$ in the column $\mathrm{A} 1$ and $\mathrm{B} 1$ respectively for the change in $\mathrm{k}_{\mathrm{sb}}$ from 15 to 100 . Both the $\mathrm{k}_{\mathrm{rs}}$ and $\mathrm{k}_{\mathrm{sb}}$ has significant influence on the columns at the first floor and the effect of $\mathrm{k}_{\mathrm{sb}}$ on moment is less if the raft is rigid $\left(\mathrm{k}_{\mathrm{rs}}=0.01\right)$. For the raft of higher rigidity and the soil of higher modulus the difference in moment between the edge columns is less.

\subsection{Bending Moment in the Beam}

In Figure 11 the variation of the support moment at B1 of beam B1-B4 is presented. The influence of $\mathrm{k}_{\mathrm{rs}}$ on the support moment is more for $\mathrm{k}_{\mathrm{rs}}=0.001$ which is evident from the increase in moment of the beam. The maximum increase in the moment due to the increase in $\mathrm{k}_{\mathrm{sb}}$ from 15 to 100 is $50 \%$ (ie 10 $\mathrm{kNm}$ to $65 \mathrm{kNm}$ ). In the rest of the beams the increase is in the range of $35 \%$ to $50 \%$. But the increase in the moment is around $10 \%$ for the increase in $\mathrm{k}_{\mathrm{sb}}$ from 15 to 30 and further increase in $\mathrm{k}_{\mathrm{sb}}$ value does not alter the support moment of the beams of all the floors in the case of $\mathrm{k}_{\mathrm{rs}}=0.01$. 


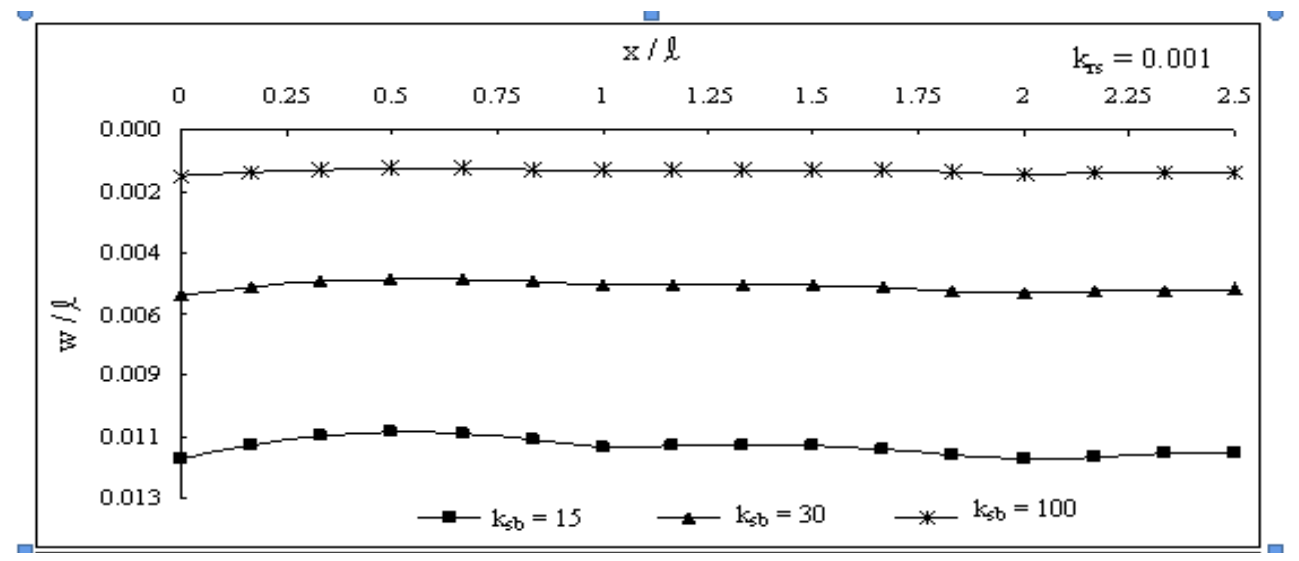

Figure 3: Normalized Settlement along the Section B1-B4 of the Raft

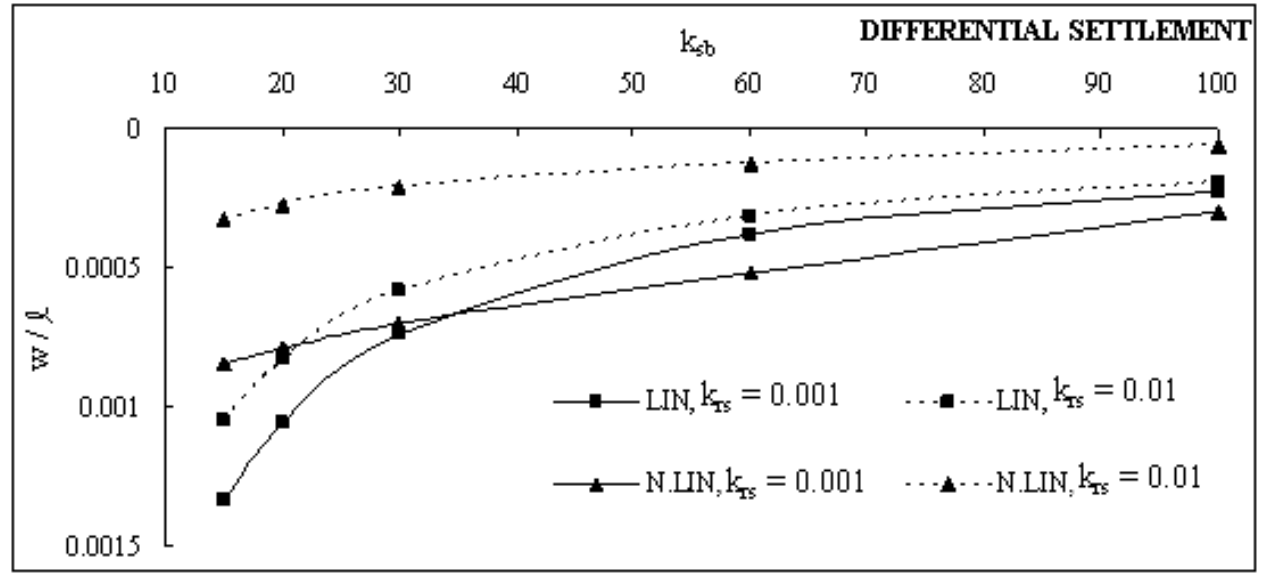

Figure 4: Variation of Differential Settlement between Linear and Non-Linear with $\mathrm{k}_{\mathrm{sb}}$ at the Corner and Centre of the Raft

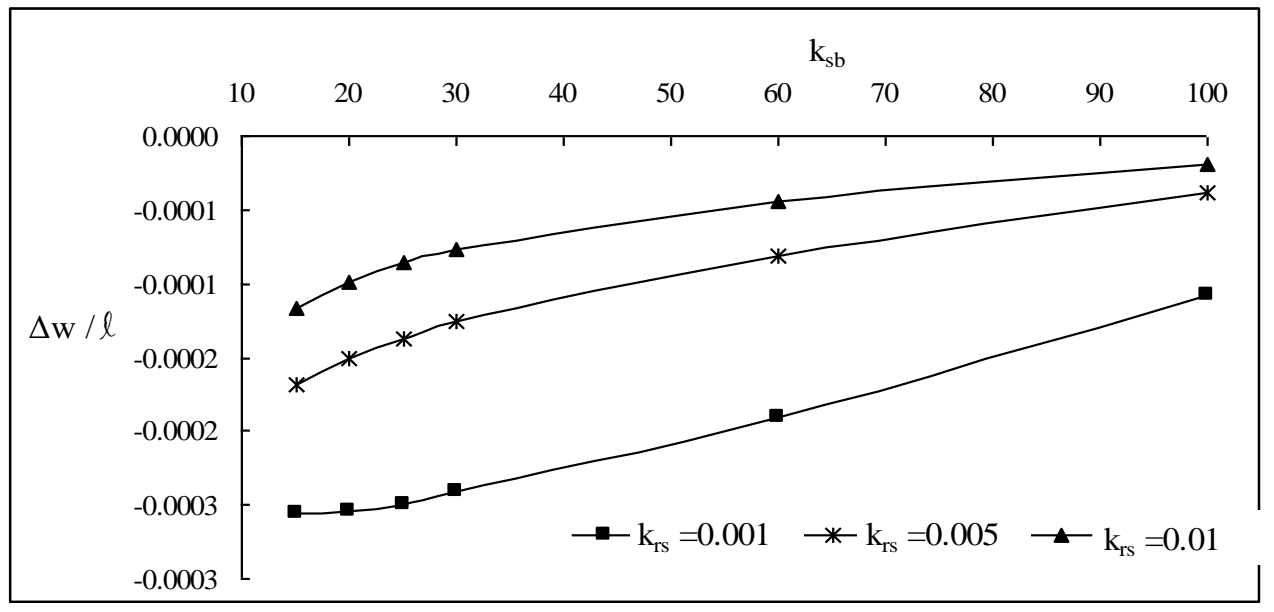

Figure 5: Effect of $\mathrm{k}_{\mathrm{sb}}$ and $\mathrm{k}_{\mathrm{rs}}$ on Differential Settlement between A1 and B3 


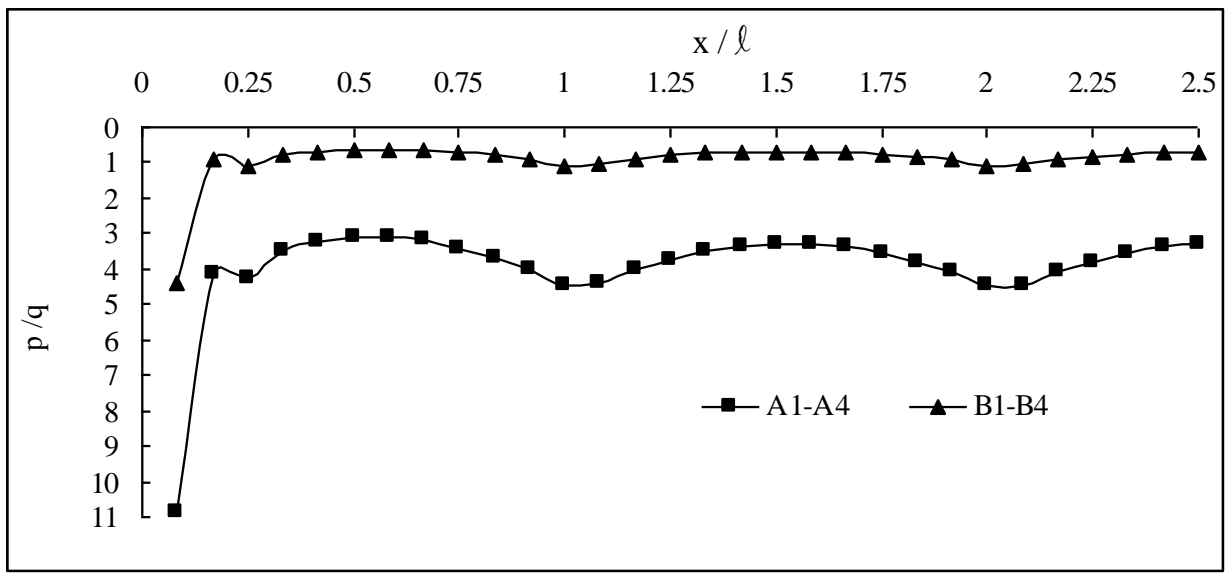

Figure 6: Contact Pressure Distribution along A1-A4 and B1-B4 (Linear)

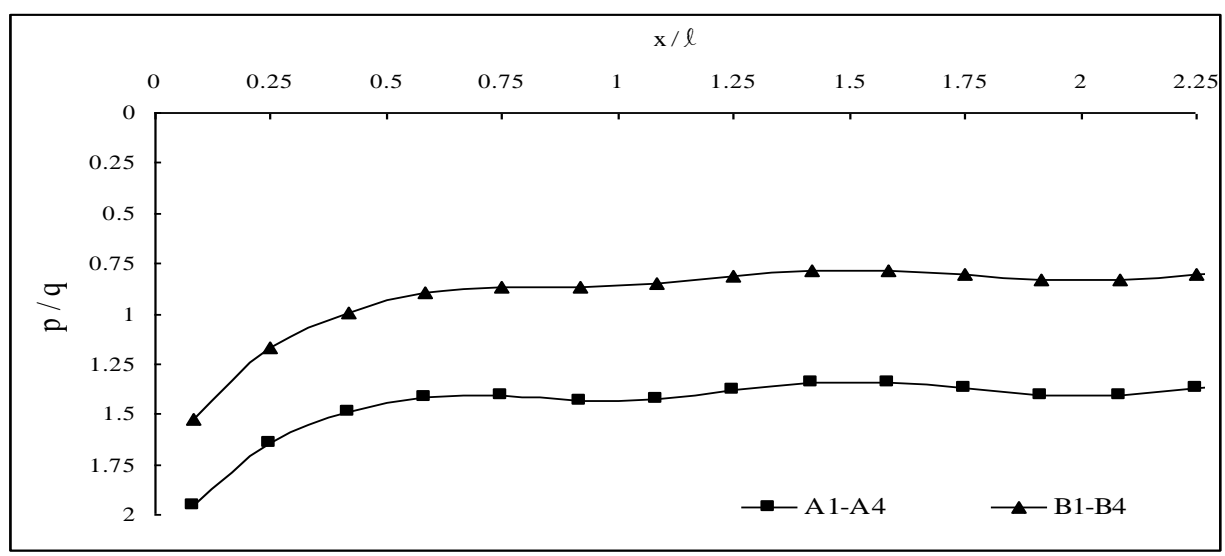

Figure 7: Contact Pressure Distribution along A1-A4 and B1-B4 (Non-linear)

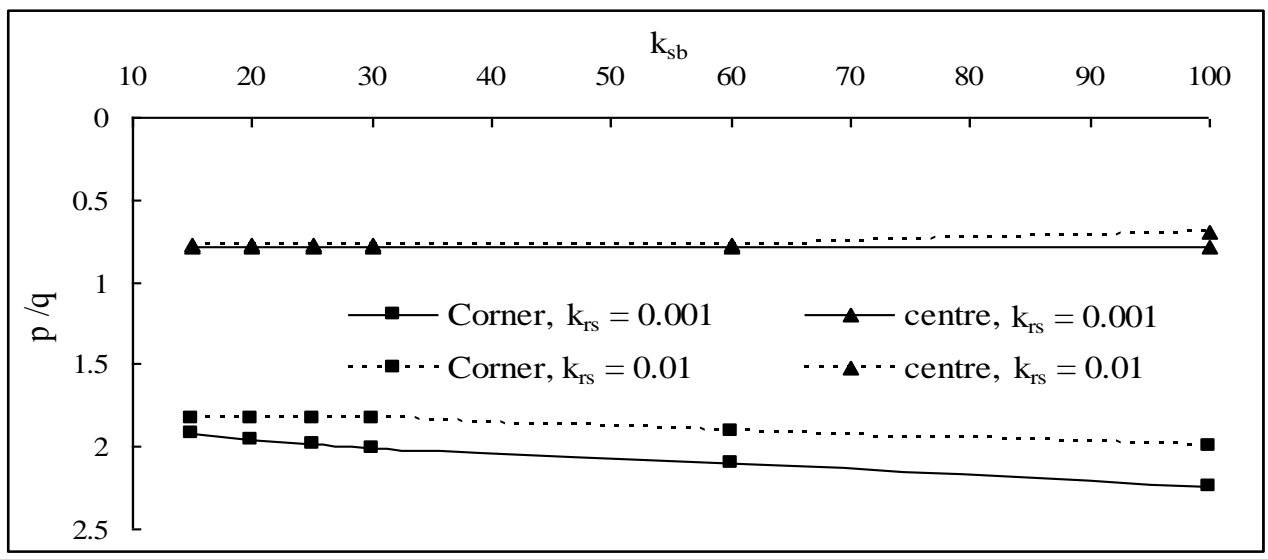

Figure 8: Variation of Contact Pressure with $\mathrm{k}_{\mathrm{sb}}$ at Corner and Centre of the Raft 


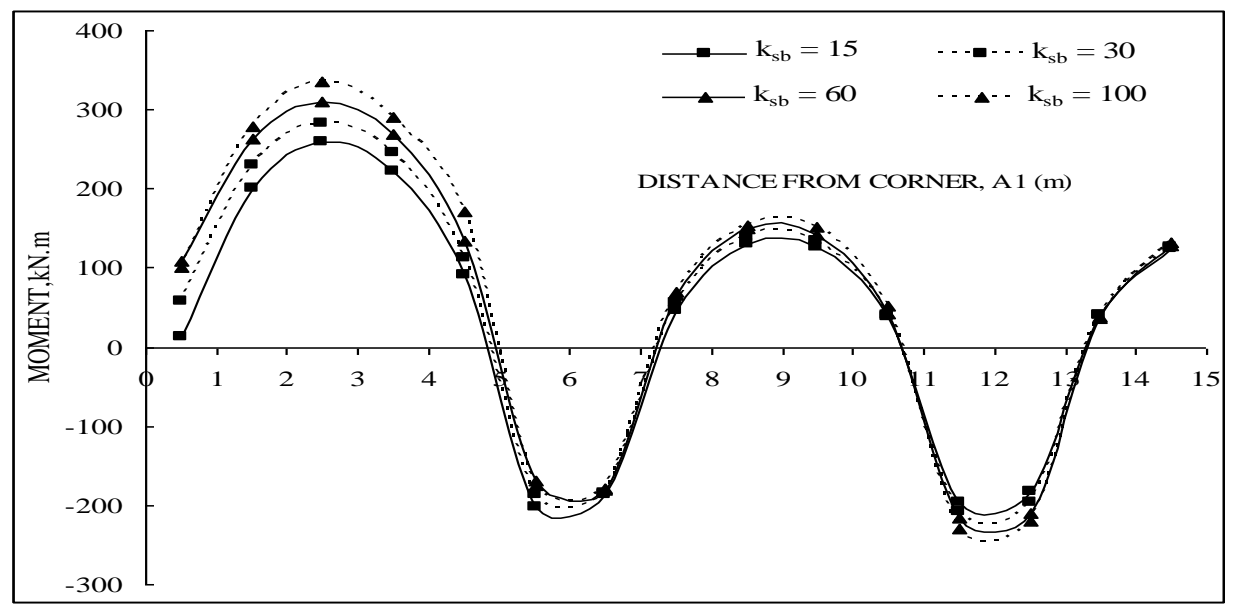

Figure 9: Variation in Bending Moment $\left(\mathrm{M}_{\mathrm{x}}\right)$ along A1-A4 of Raft

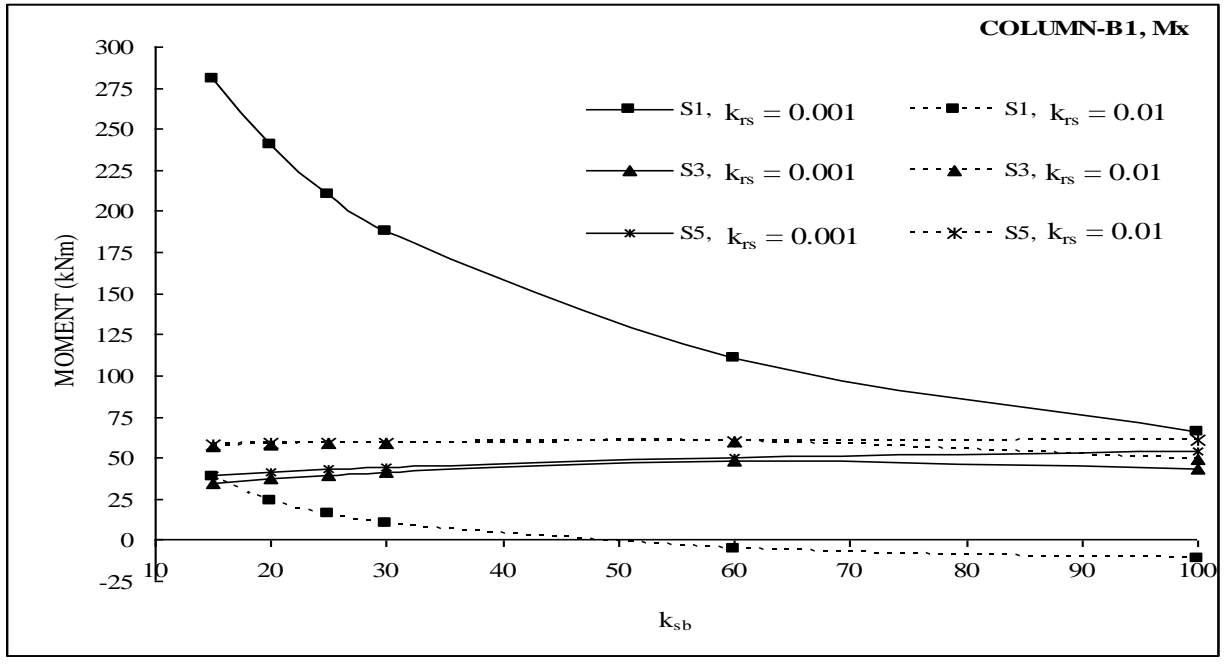

Figure 10: Variation of Column Moment B1 for Various $\mathrm{k}_{\mathrm{sb}}$ and $\mathrm{k}_{\mathrm{rs}}$

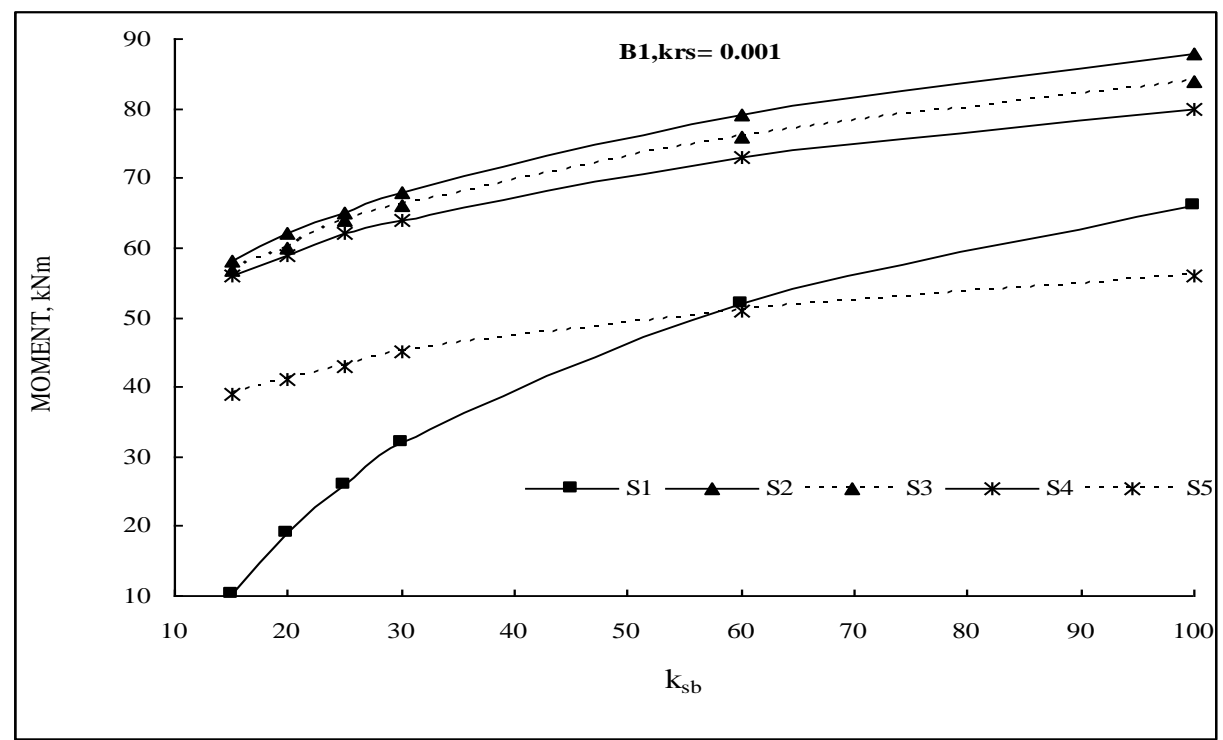

Figure 11: Variation of Beam Moment at Support B1 of beam B1-B4 


\section{CONCLUSION}

Based on the analyses of the soil- mat-space frame system, the following important conclusions are drawn.

The settlement is higher for the non-linear soil condition irrespective of $\mathrm{k}_{\mathrm{rs}}$ and $\mathrm{k}_{\mathrm{sb}}$ values. As $\mathrm{k}_{\mathrm{sb}}$ increases, the difference in the settlement decreases both at the centre and the corner of the raft and the difference become almost negligible for the higher values of $\mathrm{k}_{\mathrm{sb}}(100)$ and $\mathrm{k}_{\mathrm{rs}}(0.01)$. The decrease in the differential settlement is in the range of $63 \%$ to $80 \%$ for the $\mathrm{k}_{\mathrm{sb}}$ values between 15 and 100 . The thickness of the raft has no influence on the total settlement. But in the differential settlement the thickness of the raft has some influence.

The magnitude of the contact pressure is maximum at the corner of the raft. In the corners, the concentration of the contact pressure is around 11 times and 2 times for linear and non-linear behavior of the soil respectively. The influence of $\mathrm{k}_{\mathrm{sb}}$ on the contact pressure is almost nil. But the contact pressure decreases with the increase in $\mathrm{k}_{\mathrm{rs}}$

Bending moment in the raft, the influence of $\mathrm{k}_{\mathrm{sb}}$ is appreciable in the end bay moments. The span moments increases with an increase in $\mathrm{k}_{\mathrm{sb}}$ and the maximum increase is in the end bay

The moment in the columns at the first floor level has found to decrease with an increase in $\mathrm{k}_{\mathrm{sb}}$ for the $\mathrm{k}_{\mathrm{rs}}$ values of 0.001 and 0.01 . However, the decrease in the moment of the first floor column is high for $\mathrm{k}_{\mathrm{rs}}=0.001$ and the reduction is $65 \%$ to $70 \%$ for the change in $\mathrm{k}_{\mathrm{sb}}$ from 15 to 100 .

The influence of $\mathrm{k}_{\mathrm{rs}}$ on the beam support moment is more for $\mathrm{k}_{\mathrm{rs}}=0.001$. The maximum increase in the moment due to the increase in $\mathrm{k}_{\mathrm{sb}}$ from 15 to 100 is $50 \%$.

\section{REFERENCES}

[1] G.G. Meyerhof, 'Settlement analysis of building frames', The Structural Engineer, Pp. 369-409, 1947.

[2] M.I. orbunov Possadov and R.V. Serebrjanyi, 'Design of structures on elastic foundation', Proc. 5th Int. Conf. Soil Mechanics and Foundation Engg., Vol. 1, Pp. 643-648,1961.

[3] L.J Wardle and R.A. Fraser, 'Methods for raft foundation design including soil-structure interaction', Proc. Symp. Raft Foundations, Perth, Australia, Pp. 1-11, 1975.

[4] M. Witt 'Solutions of Plates on a Heterogeneous Elastic Foundation, Computers and Structures', Vol. 18, No. 1, Pp. 41-45,1984.

[5] Ball S.C., James S. and Notch 'Computer analysis and Design of large mat foundations', Journal of Structural Engineering, Vol.110, No.5, Pp.1180-1196, 1984.

[6] Y. Wang and J. Ni 'Plate on Non-Homogeneous elastic Half-space analysed', FEM, Structural Engg. And Mechanics, Vol. 9, No. 2, Pp. 127-139, 2000.

[7] G.J.W. King and V.S. Chandrasekaran 'Interaction analysis of rafted multistoried space frame resting on the inhomogeneous clay structure', Proc. Int. Conf. on FEM in Engineering, University of New South Wales, Pp. 492-509,1974 .

[8] R.K. Bhandari and A.R.K. Rao, 'Concept of Rigidity in foundation Analysis', International Symposium on soil structure interaction, University of Roorkee, UP, India, Pp.287-294,1977.

[9] R. Srinivasaraghavan 'Interaction among soil, foundation and superstructure', Thesis submitted for the award of Ph.D Degree, Department of Civil Engineering. IIT, Madras, 1980.
[10] Brown P.T. and Yu Si K.R., 'Load Sequence and Structure-foundationInteraction', Journal of Structural Engineering, Vol. 112, No. 3, Pp. 481488,1986 .

[11] Hora M. and Sharm A., 'Elasto-plastic analysis of building frame-soil interaction system', Journal of Structural Engineering, Vol. 34, No. 2, Pp. 124-139,2007.

[12] ANSYS Engineering Analysis System, 'User's Manual', Swanson Analysis System Inc., Houston, Pennsylvania, Version 10.

[13] Swamy, H. M. R., Krishnamoorthy, A., Prabakhara, D. L., \& Bhavikatti, S. S., Evaluation of the influence of interface elements for structure isolated footing - soil interaction analysis. Interaction and Multiscale Mechanics, an International Journal, 4(1), Pp 65-83, 2011.

[14] Zeghal. M., Edil. T.B., 'Soil Structure Interaction analysis: modeling the interface', Can.Geotech.J.39, Pp. 620-628, 2002. 